\title{
High-accuracy Photonics-assisted Frequency Measurement With Rough-accurate Compensation Based on Mach-Zehnder Interfering and Power Cancellation
}

\section{Lanfeng Huang ( $\sim$ 370689518@qq.com )}

Air Force Engineering University

Yongjun Li

Air Force Engineering University

Shanghong Zhao

Air Force Engineering University

Tao Lin

Air Force Engineering University

Guodong Wang

Air Force Engineering University

Xuan Li

Air Force Engineering University

Zihang Zhu

Air Force Engineering University

\section{Research Article}

Keywords: microwave frequency measurement, microwave photonics, Mach-Zehnder interferometer, power cancellation

Posted Date: September 28th, 2021

DOI: https://doi.org/10.21203/rs.3.rs-803615/v1

License: (c) (i) This work is licensed under a Creative Commons Attribution 4.0 International License. Read Full License 


\title{
High-accuracy photonics-assisted frequency measurement with
}

\section{rough-accurate compensation based on Mach-Zehnder interfering}

\section{and power cancellation}

\author{
Lanfeng Huang*, Yongjun Li, Shanghong Zhao, Tao Lin, Guodong Wang, Xuan Li, Zihang Zhu \\ Information and Navigation College, Air Force Engineering University, Xi'an, Shaanxi 710077, China \\ *Corresponding author: hlf.feng@qq.com
}

\begin{abstract}
A high-accuracy photonics-assisted frequency measurement with rough-accurate compensation based on Mach-Zehnder interfering and power cancellation is proposed. A polarization division multiplexing dual-parallel Mach-Zehnder modulator (PDM-DPMZM) is employed to mix the unknown RF signal and sweep signal to optical field. The rough measurement is firstly performed by the interference of a Mach-Zehnder interferometer (MZI) to realize fast frequency estimation. Then, based on the rough measurement result, the accurate measurement based on power cancellation is implemented in a much narrower range, which greatly improves the efficiency of frequency measurement. The simulation results show that the amplitude comparison function (ACF) established by interference can achieve a measurement error of less than $0.3 \mathrm{GHz}$ over $0.5 \sim 39 \mathrm{GHz}$. Moreover, thanks to the rough-accurate compensation, the accuracy can be further improved to $4 \mathrm{MHz}$. Additionally, the multiple frequency identification with a resolution of $10 \mathrm{MHz}$ can also be achieved based on this system.
\end{abstract}

Keywords: microwave frequency measurement, microwave photonics, Mach-Zehnder interferometer, power cancellation

\section{Introduction}

The frequency measurement of the intercepted microwave signals is an essential task for modern radar warning (RW) and electronic countermeasures (ECM) systems, which is often performed by specially designed receivers. The RW is required to monitor a broad frequency range threat signals in the electromagnetic space and provide real time early warning [1]. It is usually implemented by the instantaneous frequency measurement (IFM) receivers to perform a wide range, fast and medium accuracy measurement [2]. Nevertheless, the ECM needs to interfere or antiinterfere with specific frequencies, a higher accuracy is preferred. It is often performed by the scanning or channelized receivers. However, separated receivers would cause complex structures, high costs and serious electromagnetic interference. Therefore, due to the tendency of integration and miniaturization of cognitive detection system in complicated electromagnetic environment, a compact frequency measurement system is urgent desired, which can provide broad range, high accuracy and appropriate response speed measurement. However, the modern electronic-based receivers can hardly simultaneously satisfy these requirements because of the electronic bottlenecks and electromagnetic interference. Fortunately, photonic-assisted frequency measurement systems that combine the advantages of electronic and photonic techniques can overcome the limitations of electronic-based systems. 
In recent years, many photonics-assisted frequency measurement (PFM) methods have been proposed. These methods can mainly be divided into two categories: the instantaneous frequency measurement systems [3-11] and the scanning frequency measurement (SFM) systems [12-18]. The mainly principle of the instantaneous measurement system is to map the unknown radio (RF) signal frequency information to a power ratio between two electrical or optical signals. Two frequencydependent power penalty functions with complement characteristics are usually acquired through interference [3-5], dispersion [6] or polarization status [7, 8], and then the amplitude comparison function (ACF) is constructed. The measurement errors of these frequency-to-power mapping methods may reach hundreds of megahertz and only single-tone signal frequency can be measured. In order to simultaneously achieve the frequency measurement of multiple signals, the frequencyto-space method is considered [9-11]. However, large filter arrays are often required in these methods, which would downgrade the system operation and bring a large cost. Another principle to identify multiple frequencies is the scanning measurement, which is commonly performed by the frequency-to-time mapping (FTTM) method through Fourier transform [12], a recirculating frequency shifting loop [13], or a frequency scanning signal [14,15]. Additionally, due to the narrow-band filtering effect obtained by the Stimulated Brillouin scattering (SBS) can provide high frequency resolution, many methods combining the frequency scanning signal and SBS are studied [16-18]. In Ref. [16], a two-step accuracy improvement of the reference calibration and the curve smoothing is introduced to optimize the scanning measuring, and a measurement error of about $\pm 1 \mathrm{MHz}$ is achieved within 6 to $18 \mathrm{GHz}$. However, due to the scanning characteristics, the measurement response of the above methods is slow and some short-term frequency components may be missed. Therefore, the method of IFM and SFM can be employed together to achieve a better-performed frequency measurement by utilizing their complementary advantages.

In this paper, a high-accuracy photonics-assisted frequency measurement with rough-accurate compensation based on Mach-Zehnder interfering and power cancellation is proposed. Only a polarization division multiplexing dual-parallel Mach-Zehnder modulator (PDM-DPMZM) is employed to modulate the unknown RF signal and sweep signal to an optical carrier, and all the DC voltages are set to the minimum transmission point (MITP) to perform the carrier-suppressed double sideband (CS-DSB) modulation to satisfy the power cancellation condition. In this system, the rough frequency measurement (RFM) is firstly performed by the interference of a Mach-Zehnder interferometer (MZI). According to the estimated results of RFM, the scanning measurement based on power cancellation can focus on a much smaller range to acquire accurate frequency results. Thanks to the rough-accurate compensation, the proposed method can achieve a high accuracy frequency measurement with appropriate response speed over a broad range. In addition, the multifrequency identification with high resolution can also be implemented based on power cancellation.

\section{Principle of Operation and Theory}


(a)

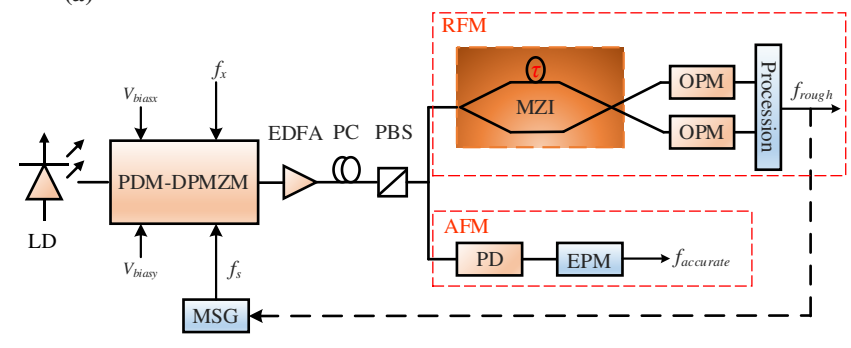

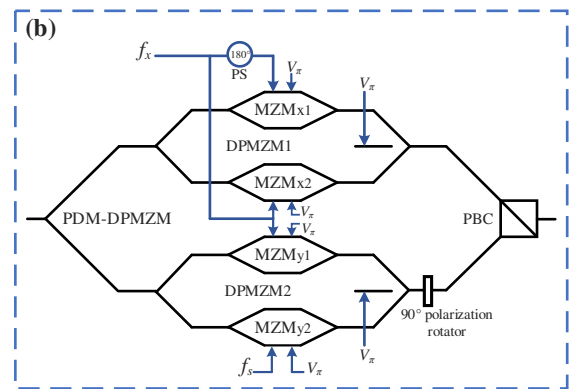

Fig. 1(a) Schematic diagram of the proposed photonics-assisted microwave frequency measurement system.

(b) The structure and signal modulation setting of PDM-DPMZM.

MSG: microwave source generator. PS: phase shifter. PC: polarization controller. PBS: polarization beam splitter. PBC: polarized beam combiner. EDFA: erbium-doped fiber amplifier. PD: photodiode. OPM: optical power meter. EPM: electric power meter.

The schematic diagram of the proposed photonics-assisted microwave frequency measurement system is shown in Fig. 1(a). The key components are a PDM-DPMZM and an MZI. A laser diode (LD) is used to generate a continuous wavelength $(\mathrm{CW})$ light $\left(E_{i n}(t)=\mathrm{E}_{\mathrm{c}} e^{j 2 \pi f_{c} t}\right)$ with the frequency of $f_{c}$ and the amplitude of $E_{c}$. This light is modulated by the unknown RF signal $f_{x}$ and the sweep signal $f_{s}$ when passing through the PDM-DPMZM. The orthogonal polarization multiplexed optical signal output from the PDM-DPMZM is power enhanced by an EDFA, and then polarization de-multiplexed into two paths ( $\mathrm{x}$ - and $\mathrm{y}$-polarizations) by the polarization beam splitter (PBS) after a polarization controller (PC). Firstly, the x-polarized light on the upper path is sent to the MZI for interference, and the output optical power of the two arms of MZI is monitored by two optical power meters for rough frequency measurement (RFM). Then the y-polarized light on the lower branch is connected to an electric power meter through the photodiode (PD) to perform accurate frequency measurement (AFM). Note that after the rough frequency measurement result is processed, the MSG is controlled to generate sweep signals in specific range, and then the accurate frequency measurement can be performed. The specific frequency measurement operation steps are shown in Table 1.

Table 1. Specific operation steps of frequency measurement.

\begin{tabular}{lll}
\hline Step 1: & The unknown RF signal is modulated and sent to the MZI to perform RFM. \\
Step 2: & Monitor the optical power output from the two arms of the MZI and calculate the related amplitude \\
RFM & comparison function (ACF) values. \\
& Step 3: & Find the related frequency from the established frequency-ACF look-up table. \\
Step 4: & Determine the scanning range of sweep signals based on the estimation result of RFM. \\
AFM & Step 5: & Monitor the electrical power after PD and record its mapping with the sweep signal $f_{s .}$ \\
& Step 6: & Find the related frequency at the power notch to determine the accurate frequency.
\end{tabular}

In the modulation section, the PDM-DPMZM is an integrated modulator that consists of two parallel sub-DPMZMs (DPMZM1 and DPMZM2), a polarization beam combiner (PBC) and a $90^{\circ}$ polarization rotator (PR) [19], as shown in Fig .1(b). Each DPMZM is composed of two sub-MZMs embedded in a main MZM. The four sub-MZMs and two main MZMs embedded in PDM-DPMZM are all biased at MITP. In the DPMZM1, the unknown RF signal is equally divided into two paths, one of the paths is introduced a $180^{\circ}$ phase shift by a phase shifter (PS) and then sent to the RF port of $\mathrm{MZM}_{\mathrm{x} 1}$. The other is directly sent to the RF port of $\mathrm{MZM}_{\mathrm{x} 2}$. Thereby the CS-DSB modulation 
can be performed. Here, the unknown RF signal can be expressed as $V_{R F}(t)=V_{R F} \sin \left(2 \pi f_{x} t\right), V_{R F}$ and $f_{x}$ are the related amplitude and frequency, respectively. On the other hand, the RF signal and sweep signal are applied to drive DPMZM2 and also implement CS-DSB modulation. The sweep signal is expressed as $V_{S}(t)=V_{S} \sin \left(2 \pi f_{s} t+\varphi\right), V_{S}, f_{s}$ and $\varphi$ are the related amplitude, frequency and initial phase respectively. Therefore, the output signal of the DPMZM1 and DPMZM2 can be expressed as:

$$
\begin{aligned}
E_{D P M Z M 1}(t)= & \frac{E_{i n}(t)}{4}\left\{\exp \left[j \frac{\pi}{2 \mathrm{~V}_{\pi}} \mathrm{V}_{\mathrm{RF}} \sin \left(2 \pi f_{x} t+\pi\right)\right]+\exp (j \pi) \exp \left[-j \frac{\pi}{2 \mathrm{~V}_{\pi}} \mathrm{V}_{\mathrm{RF}} \sin \left(2 \pi f_{x} t+\pi\right)\right]\right\} \\
& +\frac{E_{i n}(t)}{4} \exp (j \pi)\left\{\exp \left[j \frac{\pi}{2 \mathrm{~V}_{\pi}} \mathrm{V}_{\mathrm{RF}} \sin \left(2 \pi f_{x} t\right)\right]+\exp (j \pi) \exp \left[-j \frac{\pi}{2 \mathrm{~V}_{\pi}} \mathrm{V}_{\mathrm{RF}} \sin \left(2 \pi f_{x} t\right)\right]\right\} \\
E_{D P M Z M 2}(t)= & \frac{E_{i n}(t)}{4}\left\{\exp \left[j \frac{\pi}{2 \mathrm{~V}_{\pi}} \mathrm{V}_{\mathrm{RF}} \sin \left(2 \pi f_{x} t\right)\right]+\exp (j \pi) \exp \left[-j \frac{\pi}{2 \mathrm{~V}_{\pi}} \mathrm{V}_{\mathrm{RF}} \sin \left(2 \pi f_{x} t\right)\right]\right\} \\
& +\frac{E_{i n}(t)}{4} \exp (j \pi)\left\{\exp \left[j \frac{\pi}{2 \mathrm{~V}_{\pi}} \mathrm{V}_{\mathrm{S}} \sin \left(2 \pi f_{\mathrm{s}} t+\varphi\right)\right]+\exp (j \pi) \exp \left[-j \frac{\pi}{2 \mathrm{~V}_{\pi}} \mathrm{V}_{\mathrm{S}} \sin \left(2 \pi f_{\mathrm{s}} t+\varphi\right)\right]\right\}
\end{aligned}
$$

where $V_{\pi}$ is the half-wave voltage of the two DPMZMs. The DC biases of DPMZM1 and DPMZM2 are all set at $\mathrm{V}_{\pi}$ to work at MITP, which is to achieve CS-DSB modulation and is defined as power cancellation condition. In the case of small signal modulation, the sidebands higher than 1st can be neglected for the sake of low power. Therefore, the signal in Eq. (1) and Eq. (2) can be expanded as following equation after the Jacobi-Anger expansion.

$$
\begin{aligned}
E_{D P M Z M 1}(t) & =\frac{E_{i n}(t)}{4}\left[2 j \sin \left(m_{R F} \sin \left(2 \pi f_{x} t+\pi\right)\right)-2 j \sin \left(m_{R F} \sin \left(2 \pi f_{x} t\right)\right)\right] \\
& =j E_{i n}(t) J_{1}\left(m_{R F}\right)\left[\sin \left(2 \pi f_{x} t+\pi\right)-\sin \left(2 \pi f_{x} t\right)\right] \\
& =-E_{i n}(t)\left[J_{1}\left(m_{R F}\right) \exp \left(2 \pi f_{x} t\right)+J_{-1}\left(m_{R F}\right) \exp \left(-2 \pi f_{x} t\right)\right] \\
E_{D P M Z M 2}(t) & =j E_{i n}(t)\left[J_{1}\left(m_{R F}\right) \sin \left(2 \pi f_{x} t\right)-J_{1}\left(m_{S}\right) \sin \left(2 \pi f_{s} t+\varphi\right)\right]
\end{aligned}
$$

Here, $J_{n}(\bullet)$ represents the first kind of Bessel function, $m_{R F}=\pi V_{R F} / V_{\pi}$ and $m_{S}=\pi V_{S} / V_{\pi}$ are the modulation indices of the modulator. The related optical spectra output from the DPMZM1 and DPMZM2 are shown Fig. 2 (a) and Fig. 2 (b), respectively. It can be seen from Fig. 2(b-i) and Fig. 2(b-ii) that power cancellation can be achieved when $f_{s}=f_{x}$.

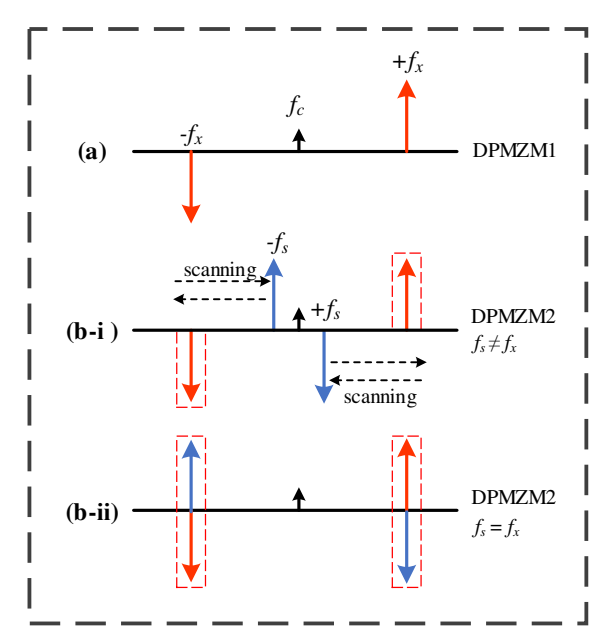


Fig. 2(a) The spectra of optical signal output from DPMZM1. (b-i) and (b-ii) The diagram of power cancellation principle.

\subsection{Rough frequency measurement}

The rough frequency measurement is implemented by the interference of an MZI, which can instantaneously estimate the unknown RF signal in a wide operation range. This method exploits the complementary transmission characteristics of the MZI. It is realized by propagating the $\mathrm{x}$ polarized CS-DSB optical signal through the MZI, where the two arms of the MZI have different power responses. The amplitude comparison between the monitored optical powers of the two output ports can be established to estimate the unknown signal frequency.

Ignoring the loss of each arm of the MZI, the optical signals at the two output ports of the MZI can be given by

$$
\begin{aligned}
& {\left[\begin{array}{l}
E_{1}(t) \\
E_{2}(t)
\end{array}\right]=\frac{1}{2}\left[\begin{array}{ll}
1 & j \\
j & 1
\end{array}\right]\left[\begin{array}{cc}
e^{j \phi_{1}} & 0 \\
0 & e^{j \phi_{2}}
\end{array}\right]\left[\begin{array}{cc}
1 & j \\
j & 1
\end{array}\right]\left[\begin{array}{c}
E_{\mathrm{DPMZM} 1}(t) \\
0
\end{array}\right]=\frac{1}{2}\left[\begin{array}{l}
E_{\mathrm{DPMZM} 1}(t) e^{j \phi_{1}}-E_{\mathrm{DPMZM} 1}(t) e^{j \phi_{2}} \\
j E_{\mathrm{DPMZM} 1}(t) e^{j \phi_{1}}+j E_{\mathrm{DPMZM} 1}(t) e^{j \phi_{2}}
\end{array}\right]} \\
& =\frac{1}{2} \mathrm{E}_{0} J_{1}(m)\left[\begin{array}{l}
e^{j 2 \pi\left(f_{c}-f_{x}\right) t} e^{j \frac{n \pi\left(f_{c}-f_{x}\right)\left(L_{1}+L_{2}\right)}{-c}} \sin \left(\pi\left(f_{c}-f_{x}\right) \tau\right)+e^{j 2 \pi\left(f_{c}+f_{x}\right) t} e^{j \frac{n \pi\left(f_{c}+f_{x}\right)\left(L_{1}+L_{2}\right)}{-c}} \sin \left(\pi\left(f_{c}+f_{x}\right) \tau\right) \\
j e^{j 2 \pi\left(f_{c}-f_{x}\right) t} e^{j \frac{n \pi\left(f_{c}-f_{x}\right)\left(L_{1}+L_{2}\right)}{-c}} \cos \left(\pi\left(f_{c}-f_{x}\right) \tau\right)+j e^{j 2 \pi\left(f_{c}+f_{x}\right) t} e^{j \frac{n \pi\left(f_{c}+f_{x}\right)\left(L_{1}+L_{2}\right)}{-c}} \cos \left(\pi\left(f_{c}+f_{x}\right) \tau\right)
\end{array}\right]
\end{aligned}
$$

Here, $\Phi_{i}=-2 \pi f_{i} n L_{i} / c,(i=1,2)$ are the phase shift that induced by the optical paths' lengths $\left(L_{1}\right.$ and $\left.L_{2}\right)$ in the two arms of MZI. $\tau$ denotes the time delay induced by the path length difference of the MZI, which can be expressed as $\tau=n\left(L_{1}-L_{2}\right) / c . n$ and $c$ are the refractive index and the speed of light in vacuum, respectively. As a result, the related optical powers can be expressed as

$$
\begin{aligned}
P_{1} & \propto \mathrm{E}_{0}^{2} J_{1}^{2}(m)\left\{\sin ^{2}\left[\pi\left(f_{c}-f_{x}\right) \tau\right]+\sin ^{2}\left[\pi\left(f_{c}+f_{x}\right) \tau\right]\right\} \\
& =\mathrm{E}_{0}^{2} J_{1}^{2}(m)\left[1-\cos \left(2 \pi f_{c} \tau\right) \cos \left(2 \pi f_{x} \tau\right)\right] \\
P_{2} & \propto \mathrm{E}_{0}^{2} J_{1}^{2}(m)\left[1+\cos \left(2 \pi f_{c} \tau\right) \cos \left(2 \pi f_{x} \tau\right)\right]
\end{aligned}
$$

Therefore, the amplitude comparison function can be constructed as

$$
A C F=\frac{P_{1}}{P_{2}}=\frac{1-\cos \left(2 \pi f_{c} \tau\right) \cos \left(2 \pi f_{x} \tau\right)}{1+\cos \left(2 \pi f_{c} \tau\right) \cos \left(2 \pi f_{x} \tau\right)}
$$

Obviously, since ACF is the power ratio of two optical signals, the influence of power fluctuation (including optical power and microwave power) caused by the system link on the measurement accuracy can be eliminated. It depends on the time delay $\tau$, the optical carrier frequency $f_{c}$ and the unknown signal frequency $f_{x}$. When the time delay of MZI is fixed, the optical carrier frequency is set at the peak or notch of the transmission response of MZI as shown in Fig. 3(a), and the simulated optical power and calculated ACF of its two output ports are shown in Fig. 3(b). A monotone region of ACF is $1 / 2 \tau$, in this range the relationship between ACF and microwave frequency is unique. To achieve higher resolution measurement, the first monotone interval of ACF is selected to establish the ACF-frequency look-up table, and the unknown signal frequency is extracted from the measured ACF. 

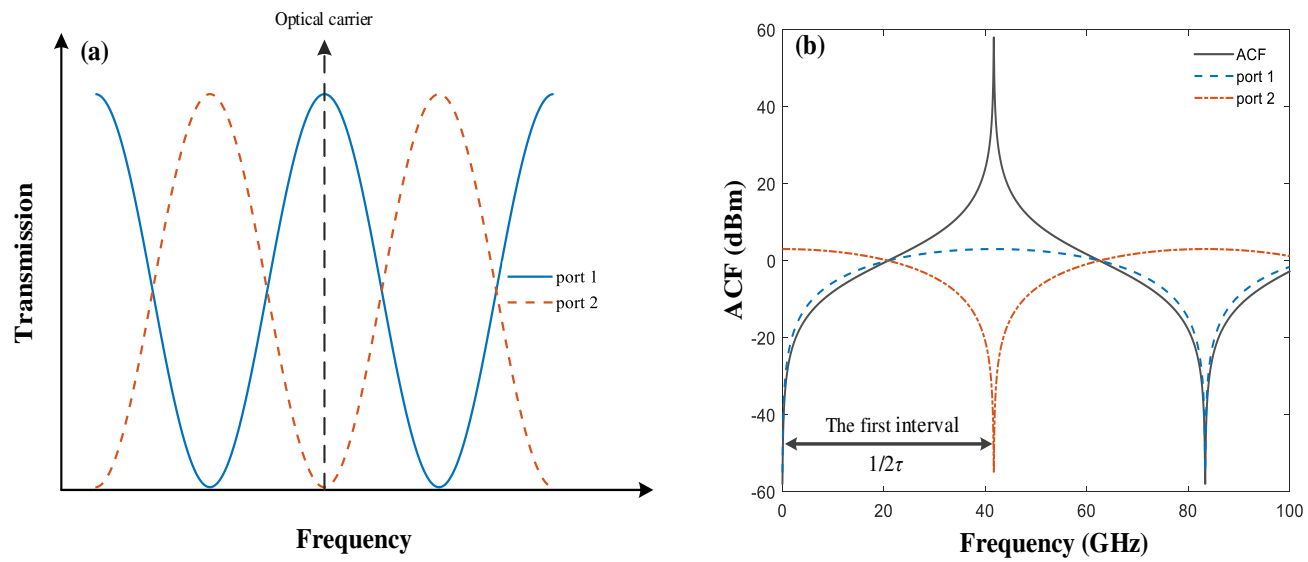

Fig. 3. (a) Schematic diagram of the transmission response of MZI. (b) Simulated output optical power at the two output ports of MZI and the related calculated ACF.

\subsection{Accurate frequency measurement}

The tradeoff between measurement range and measurement accuracy is inevitable, which is a great challenge to the ACF constructed by the frequency-to-power mapping method. A large measurement range will lead to a decrease in the slope of ACF, thereby deteriorating the measurement accuracy. In applications, the measurement range and accuracy are often compromised according to actual requirements. Therefore, we propose a frequency measurement method based on rough-accurate compensation to overcome this problem. Noteworthy, to quickly judge which band is the received signal located and greatly lessen the sweeping range, the rough estimation should cover a wide frequency monitoring range. In this case, the accuracy can be compromised to a medium level. Then, based on the roughly judged band, the accurate frequency measurement can be implemented to accurately extract out the signal frequency.

To realize accurate frequency measurement, power cancellation is performed by frequency sweeping. Thanks to the results of rough frequency measurement, the unknown RF signal frequency has been positioned in a narrow range, which means we can scan a much narrower band to quickly extract out the precise frequency. For example, the unknown signal with the frequency of $f_{x}$ is estimated by the RFM as the approximate frequency of $f_{\text {rough }}$. Here, the measurement error of RFM is $\Delta \mathrm{err}= \pm\left|f_{\text {rough }}-f_{x}\right|$. It can be easily known that $f_{x}$ locates in the range of $\left[f_{\text {rough }}-\Delta \mathrm{err}, f_{\text {rough }}+\Delta \mathrm{err}\right]$. Therefore, only scanning the $\left[f_{\text {rough }}-\Delta \mathrm{err}, f_{\text {rough }}+\Delta \mathrm{err}\right]$ is enough, which can greatly improve the speed of accurate measurement. It should be strengthened that $\Delta$ err is previously known. We can previously test the rough estimation section to find the maximum error Errormax, which can be regarded as $\Delta$ err.

The optical signal modulated with the unknown signal and sweep signal is injected into an EDFA with a gain of $G$. Then it is sent to a photodiode. For further analysis, we assume that the amplitude of the sweep signal is $k$ times that of the unknown RF signal (i.e. $V_{S}=k V_{R F}$ ). When the driven signal power is low to satisfy the small signal modulation, $J_{1}\left(m_{R F}\right) \approx m_{R F} / 2, J_{1}\left(m_{S}\right) \approx m_{S} / 2=\mathrm{k} m_{R F}$ /2. Therefore, the detected current related to Eq. (4) can be written as 


$$
\begin{aligned}
I(t) & =\Re G^{2} E_{\text {DPMZM } 2}(t) E_{D P M Z M 2}^{*}(t) \\
& =\Re G^{2} E_{c}^{2}\left[\begin{array}{l}
\left.J_{1}^{2}\left(m_{R F}\right) \sin ^{2}\left(2 \pi f_{x} t\right)+J_{1}^{2}\left(m_{S}\right) \sin ^{2}\left(2 \pi f_{s} t+\varphi\right)\right] \\
-2 J_{1}\left(m_{R F}\right) J_{1}\left(m_{S}\right) \sin \left(2 \pi f_{x} t\right) \sin \left(2 \pi f_{s} t+\varphi\right)
\end{array}\right] \\
& =\frac{1}{2} \Re G^{2} E_{c}^{2}\left\{\begin{array}{l}
J_{1}^{2}\left(m_{R F}\right)\left[1-\cos \left(4 \pi f_{x} t\right)\right]+J_{1}^{2}\left(m_{S}\right)\left[1-\cos \left(4 \pi f_{s} t+2 \varphi\right)\right] \\
+2 J_{1}\left(m_{R F}\right) J_{1}\left(m_{S}\right)\left[\cos \left(2 \pi\left(f_{x}+f_{s}\right) t+\varphi\right)-\cos \left(2 \pi\left(f_{x}-f_{s}\right) t-\varphi\right)\right]
\end{array}\right\} \\
& =\frac{1}{8} \Re G^{2} E_{c}^{2} m_{R F}^{2}\left\{\begin{array}{l}
{\left[1-\cos \left(4 \pi f_{x} t\right)\right]+k^{2}\left[1-\cos \left(4 \pi f_{s} t+2 \varphi\right)\right]} \\
+2 k\left[\cos \left(2 \pi\left(f_{x}+f_{s}\right) t+\varphi\right)-\cos \left(2 \pi\left(f_{x}-f_{s}\right) t-\varphi\right)\right]
\end{array}\right\}
\end{aligned}
$$

where $\mathfrak{R}$ is the responsivity of the photodiode. It can be found that the detected current contains DC components, second-order components and the converted components. Therefore, the related electric power can be expressed as

$$
\begin{aligned}
P & =\frac{1}{2} \Re^{2} G^{4} E_{c}^{4}\left[J_{1}^{4}\left(m_{R F}\right)+J_{1}^{4}\left(m_{S}\right)+5 J_{1}^{2}\left(m_{R F}\right) J_{1}^{2}\left(m_{S}\right)\right] \\
& =\frac{1}{32} \Re^{2} G^{4} E_{c}^{4} m_{R F}^{4}\left(k^{4}+5 k^{2}+1\right)
\end{aligned}
$$

There are two cases when scanning the sweep signal frequency, and the related spectra are shown in Fig. 2(b-i) and Fig. 2(b-ii). As shown in Fig. 2(b-i), the sweep frequency is usually misaligned with the unknown RF frequency (i.e. $f_{s} \neq f_{x}$ ), which does not meet the power cancellation condition. However, a special case should be noted. As shown in Fig. 2(b-ii), when $f_{s}=f_{x}$, the sideband stimulated by the unknown RF signal will be weakened or even cancelled. In this special case, the optical signal output from DPMZM2 and the related detected current can be written as

$$
\begin{aligned}
\left.E_{D P M Z M 2}(t)\right|_{f_{s}=f_{x}} & =j E_{\text {in }}(t)\left[J_{1}\left(m_{R F}\right) \sin \left(2 \pi f_{x} t\right)-J_{1}\left(m_{S}\right) \sin \left(2 \pi f_{x} t+\varphi\right)\right] \\
& =\frac{j E_{\text {in }}(t) m_{R F}}{2}\left[\sin \left(2 \pi f_{x} t\right)-k \sin \left(2 \pi f_{x} t+\varphi\right)\right] \\
& =\frac{E_{\text {in }}(t) m_{R F}}{4}\left[\exp \left(j 2 \pi f_{x} t\right)-\exp \left(-j 2 \pi f_{x} t\right)-k \exp \left(j 2 \pi f_{x} t+j \varphi\right)+k \exp \left(-j 2 \pi f_{x} t-j \varphi\right)\right] \\
& =\frac{E_{i n}(t) m_{R F}}{4}\left\{\exp \left(j 2 \pi f_{x} t\right)[1-k \exp (j \varphi)]-\exp \left(-j 2 \pi f_{x} t\right)[1-k \exp (-j \varphi)]\right\}
\end{aligned}
$$$$
\begin{aligned}
\left.I(t)\right|_{f_{s}=f_{x}} & =\frac{1}{8} \mathfrak{R} G^{2} E_{c}^{2} m_{R F}^{2}\left\{\begin{array}{l}
{\left[1-\cos \left(4 \pi f_{x} t\right)\right]+k^{2}\left[1-\cos \left(4 \pi f_{x} t+2 \varphi\right)\right]} \\
+2 k\left[\cos \left(2 \pi\left(f_{x}+f_{x}\right) t+\varphi\right)-\cos \left(2 \pi\left(f_{x}-f_{x}\right) t-\varphi\right)\right]
\end{array}\right\} \\
& =\frac{1}{8} \Re G^{2} E_{c}^{2} m_{R F}^{2}\left\{\begin{array}{l}
{\left[1-\cos \left(4 \pi f_{x} t\right)\right]+k^{2}\left[1-\cos \left(4 \pi f_{x} t+2 \varphi\right)\right]} \\
+2 k\left[\cos \left(4 \pi f_{x} t+\varphi\right)-\cos \varphi\right]
\end{array}\right\}
\end{aligned}
$$

Comparing Eq. (8) with Eq. (11), it can be found that when $f_{s}=f_{x}$, the converted frequency component $f_{s}-f_{x}$ has been total cancelled, resulting in a reduction of the related power. For convenience of calculation, Eq. (11) can be written as 


$$
\begin{aligned}
\left.I(t)\right|_{f_{s}=f_{x}}=\frac{\Re G^{2} E_{c}^{2} m_{R F}^{2}}{16}\left\{\begin{array}{l}
\exp \left(j 2 \pi f_{x} t\right)[1-k \exp (j \varphi)]-\exp \left(-j 2 \pi f_{x} t\right)[1-k \exp (-j \varphi)] \\
\times\left\{\exp \left(-j 2 \pi f_{x} t\right)[1-k \exp (-j \varphi)]-\exp \left(j 2 \pi f_{x} t\right)[1-k \exp (j \varphi)]\right\}
\end{array}\right\} \\
=\frac{\Re G^{2} E_{c}^{2} m_{R F}^{2}}{16}\left\{\begin{array}{l}
2\left[1-k \exp (j \varphi)-k \exp (-j \varphi)+k^{2}\right] \\
-\exp \left(j 4 \pi f_{x} t\right) \underbrace{\left[1-2 k \exp (j \varphi)+k^{2} \exp (j 2 \varphi)\right]}_{A(D C)} \\
-\exp \left(-j 4 \pi f_{x} t\right) \underbrace{\left[1-2 k \exp (-j \varphi)+k^{2} \exp (-j 2 \varphi)\right]}_{A\left(2 f_{x}\right)}
\end{array}\right\}
\end{aligned}
$$

And the related electric power in this special case can be written as

$$
\begin{aligned}
\left.P\right|_{f_{s}=f_{x}} & \propto \Re^{2} G^{4} E_{c}^{4} m_{R F}^{2}\{\underbrace{4\left(k^{2}-2 k \cos \varphi+1\right)^{2}}_{P(D C)}+\underbrace{[k \exp (j \varphi)-1]^{4}}_{P\left(2 f_{x}\right)}+\underbrace{[k \exp (-j \varphi)-1]^{4}}_{P\left(-2 f_{x}\right)}\} \\
& =\mathfrak{R}^{2} G^{4} E_{c}^{4} m_{R F}^{2}\left\{\left[4(k \cos \varphi-1)^{2}-2\left(k^{2}-2 k \cos \varphi+1\right)\right]^{2}+2\left(k^{2}-2 k \cos \varphi+1\right)^{2}\right\}
\end{aligned}
$$

Here, $A(D C)$ represents the amplitude of the DC (direct current) component, $A\left(2 f_{x}\right)$ and $\mathrm{A}\left(-2 f_{x}\right)$ are the amplitude of the converted frequency component $2 f_{x}$ and $-2 f_{x}$, respectively. Obviously, when the sweep signal frequency overlaps with the unknown RF signal, the total power is different. Only the DC and the frequency components of $2 f_{x}$ and $-2 f_{x}$ are remained. Therefore, the power notches can be achieved.

\section{Simulation demonstration}

To verify the feasibility of the proposed photonics-assisted frequency measurement system, a simulation work based on the structure shown in Fig. 1 is conducted through the software "OptiSystem". In this work, the frequency, power and linewidth of the optical carrier generated by the $\mathrm{LD}$ are set to $193.0833 \mathrm{THz}, 15 \mathrm{dBm}$ and $1 \mathrm{MHz}$, respectively. The power of the unknown RF signal is $7 \mathrm{dBm}$, the gains of EDFA are $20 \mathrm{~dB}$. The time delay $\tau$ of the MZI is set to $12 \mathrm{ps}$.

The simulation results of rough frequency measurement are shown in Fig. 4. We have monitored the optical power output from the two ports of MZI with different input RF frequencies. And the measured optical power responses are shown in Fig. 4(a). As predicted by theory, the power of two optical signals after interference is complementary. Meanwhile, the calculated ACF agrees well with the theoretical prediction of Eq. (7). Thus, an ACF-frequency look-up table can be established to achieve RFM. As can be seen from Fig. 4(b), in the range of $0.5 \mathrm{GHz}-39 \mathrm{GHz}$, the absolute frequency measurement error calculated by comparing the estimated frequency and the actual input frequency is less than $0.3 \mathrm{GHz}$. It also means the maximum error Error $_{\max }=0.3 \mathrm{GHz}$. 

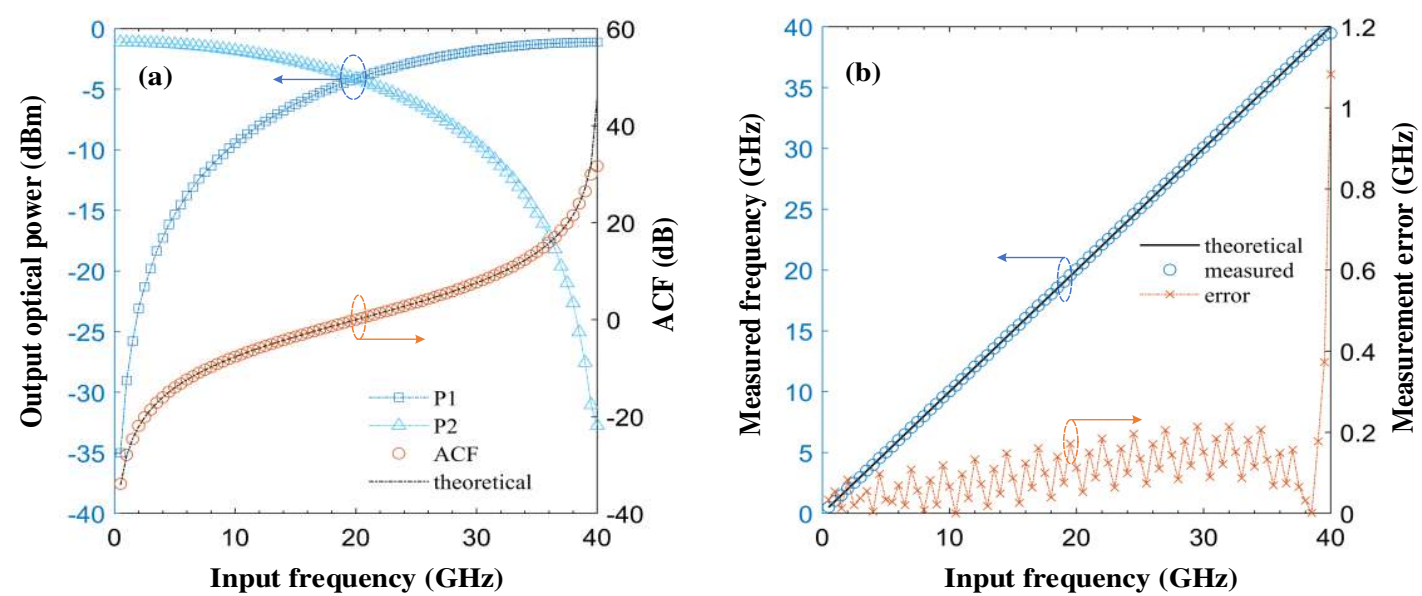

Fig. 4. Simulation measurement results of the RFM. (a) Monitored optical power at the two output ports of the MZI, and the calculated ACF. (b) The absolute frequency measurement error between the estimated frequency and the actual input frequency.

Notedly, two conditions for the ideal case of power cancellation are defined firstly in the AFM: (1) The amplitude of the unknown signal is equal to the sweep signal, i.e. the amplitude ratio $k=1$. (2) The phase difference between the unknown signal and the sweep signal is zero, i.e. the sweep signal's initial phase $\varphi=0$. In this work, the ideal case of power cancellation is firstly considered to demonstrate the feasibility of frequency measurement. Then more common and complex scenarios are discussed in the following "Discussion" section.

According to the results of RFM before, the frequency of the unknown RF signal can be approximately estimated as $f_{\text {rough }}$, and the maximum absolute measurement error $\Delta \mathrm{err}$ is $0.3 \mathrm{GHz}$. Therefore, the AFM can be implemented by power cancellation within the range of $\left[f_{\text {rough }}-\Delta \mathrm{err}\right.$, $\left.f_{\text {rough }}+\Delta \mathrm{err}\right]$. For example, when the actual frequency of the unknown RF signal is $5 \mathrm{GHz}$, scanning the sweep signal frequency from $4.7 \mathrm{GHz}$ to $5.3 \mathrm{GHz}$, with the step of $0.001 \mathrm{GHz}$. The monitored results are shown in Fig. 5(a)-(b). Obviously, when the sweep signal frequency overlaps with the unknown RF signal, (i.e. $f_{s} \approx f_{x}$ ) the sidebands stimulated by the RF signal are excluded, resulting in a power notch. In the ideal case of power cancellation, a power notch with cancellation ratio (CR) of $23 \mathrm{~dB}$ can be acquired. In the theoretical analysis, only when the frequency of the sweep signal is strictly equal to that of the unknown RF signal (i.e. $f_{s}=f_{x}$ ), can the power notch be achieved. However, due to the influence of the linewidth of the optical carrier and the noise in the link, the width of the power notch is broadened to $4 \mathrm{MHz}$. The measured results of AFM are shown in Fig. 5(c). It can be clearly seen that the input RF frequency is highly consistent with the measured frequency in the range of $0.5 \mathrm{GHz}$ to $39 \mathrm{GHz}$, and the measurement error is within $4 \mathrm{MHz}$. Compared with the measurement results of RFM, the accuracy is significantly improved. 

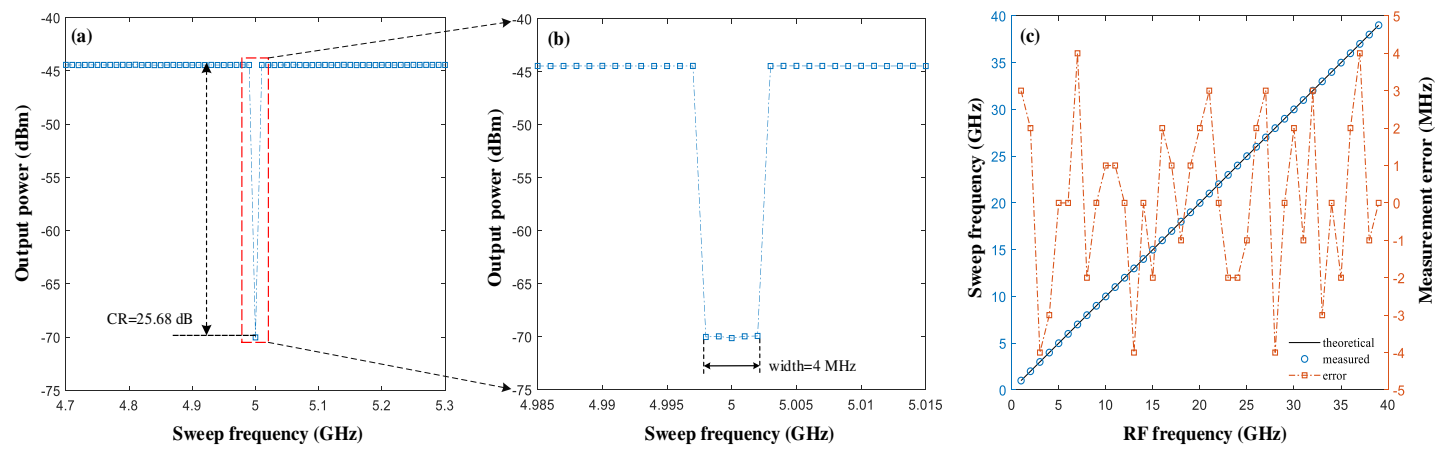

Fig.5. (a) The monitored sweeping results. (b) The zoom in figure of the measured result of the unknown RF signal. (c)The measured results and errors of AFM.

\section{Discussion}

\subsection{The influence of the sideband to carrier ratio (SCR) on RFM accuracy.}

In the rough frequency measurement, the accuracy is mainly influenced by the SCR of the CSDSB signal injected into MZI. In theory, the optical carrier is set at the notch or peak of the transmission of MZI, and completely suppressed when output from DPMZM1. Therefore, the power of the optical signal input MZI is mainly concentrated in the first-order sidebands, which carries the frequency information of the unknown RF signal. However, the residual carrier may exist due to the non-ideal extinction ratio of the modulator. Moreover, although the residual carrier is completely suppressed on the path $\mathrm{P}_{\text {notch }}$ of MZI (at the notch), the residual carrier on another path $\mathrm{P}_{\text {peak }}$ (at the peak) is strengthen, which occupies partial power of the output signal of MZI and makes the measured output power deviate from the theoretical value. Therefore, the influence of the residual carrier mainly comes from $P_{\text {peak. }}$. Here, we concentrate on the output results from $P_{\text {peak }}$ to investigate the influence of residual carrier on the accuracy of RFM.

The measurement results of RFM with different SCRs are shown in Fig. 6(a). As the SCR of the CS-DSB signal injected into MZI varies from $15 \mathrm{~dB}$ to $30 \mathrm{~dB}$. It is clear that the measured results agree well with the theoretical values in the range below $32 \mathrm{GHz}$. When the frequency exceeds 32 $\mathrm{GHz}$, the measured results gradually deviate from the theoretical values, and the deviation decreases with the increase of SCR. Besides, when the SCR exceeds $25 \mathrm{~dB}$, the measurement error below 0.4 $\mathrm{GHz}$ can be achieved within $0.5 \mathrm{GHz}-39 \mathrm{GHz}$. The reasons for the deterioration of the measurement accuracy in the high frequency part are as follows:

(1) The residual carrier on the path $P_{\text {peak }}$ of MZI is enhanced, accounting for partial power of the output signal after interference; (2) The 1st-order sidebands intensity on the $\mathrm{P}_{\text {peak }}$ are reduced as the increase of the RF signal frequency, resulting in a gradual decrease in the SCR of the interfered output signal. As a result, the residual carrier occupies the major power of the output signal, which can hardly be ignored. In this case, the transmission responses of the two paths of MZI no longer have complementary characteristics, and the measured ACF gradually deviates from the theoretical curve in the high frequency portion. For a more intuitive demonstration, when SCR of the CS-DSB signal injected into MZI is $20 \mathrm{~dB}$, the output signal spectra after interference on $P_{\text {peak }}$ is shown in Fig. 6 (b). When the RF signal frequency is $5 \mathrm{GHz}$ and $20 \mathrm{GHz}$, the SCR of the interfered output signal is $19.78 \mathrm{~dB}$ and $16.97 \mathrm{~dB}$, respectively. While the RF frequency increases to $39 \mathrm{GHz}$, the SCR deteriorates to $-7.93 \mathrm{~dB}$ dramatically. Therefore, in applications, the carrier should be excluded as much as possible to ensure that the SCR of the signal input MZI is better than $25 \mathrm{~dB}$, so that a 
well-performed high frequency ( $>32 \mathrm{GHz}$ ) measurement can be achieved. Fortunately, in the proposed modulation scheme, the main modulator of DPMZM1 is biased at MITP, which reverses and excludes the optical carrier output of two parallel sub-MZMs, thus ensuring a high SCR.
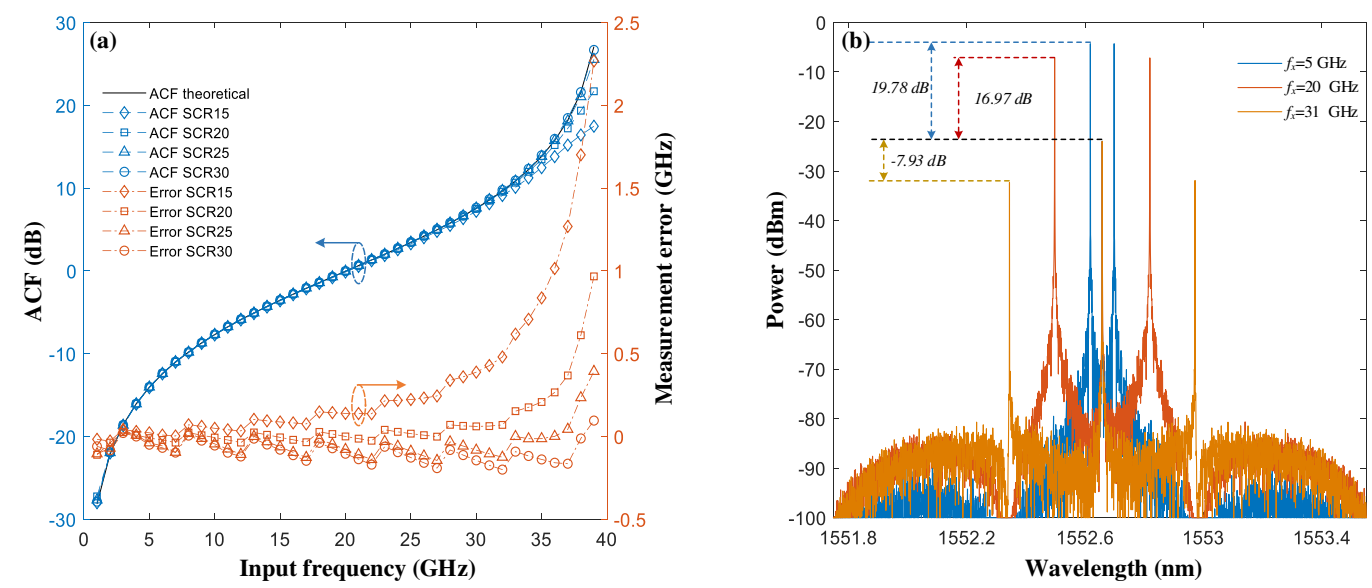

Fig. 6. Analysis of the influence of SCR on RFM accuracy. (a) Measured ACF responses and errors with different SCRs. (b) The output signal spectra after interference on the path of $\mathrm{P}_{\text {peak. }}$

\subsection{The influence of amplitude ratio and initial phase on the AFM.}

In the last section, we demonstrate the feasibility of AFM in the ideal cases of power cancellation. However, in an actual electronic warfare, the amplitude and phase of the intercepted RF signal are uncertain. In order to make it suitable for a real detection situation, the influence of amplitude ratio $k$ and initial phase $\varphi$ on the AFM are discussed.

According to the theoretical derivation of Eq. (13), at the notch of power cancellation (i.e. $f_{s}=f_{x}$ ), the power is associated with the amplitude ratio $k$ and the initial phase $\varphi$. Their theoretical relationship and contour are shown in Fig. 7(a). It can be seen that the power is positively corrected with the amplitude ratio $k$ and the initial phase $\varphi$. Obviously, around the ideal case of power cancellation, the power is the lowest and the variation is the sharpest. In practical applications, the depth of power notch (i.e. power cancellation ratio $\mathrm{CR}$ ) affects the quality of the measurement. Firstly, in this system, $\mathrm{CR}=3 \mathrm{~dB}$ is defined as the lowest distinguishing boundary (LDB). If CR is lower than $3 \mathrm{~dB}$, the power notch stimulated by this RF frequency cannot be well distinguished. The contour of CR is shown in Fig.7 (b). In theory, a distinguishable power notch can be acquired in a region where the contour value exceeds $3 \mathrm{~dB}$. To verify the correctness of theoretical analysis, the following simulation studies the influence of $k$ and $\varphi$ on CR separately. As shown in Fig. 7(c), when the $\varphi=0, \mathrm{CR}$ decreases with the increase of $k$. When $k$ increases to 2.3, CR reaches the LDB. In other words, when the sweep signal power is $7.234 \mathrm{~dB}$ higher than the unknown RF signal, the power cannot be well distinguished. Similarly, when $k=1, \mathrm{CR}$ decreases with the increase of $\varphi$, and the LDB is about 1.5. It can be seen that the simulation results of Fig. 7(c) and Fig. 7(d) are almost consistent with the theoretical prediction of Fig. 7(b). In the application, in order to obtain a wellperformed distinguishable power notch, $k=1$ should be guaranteed as much as possible. Moreover, if a leak detection occurs, adjust $\varphi$ and rescan until the notch appears. 

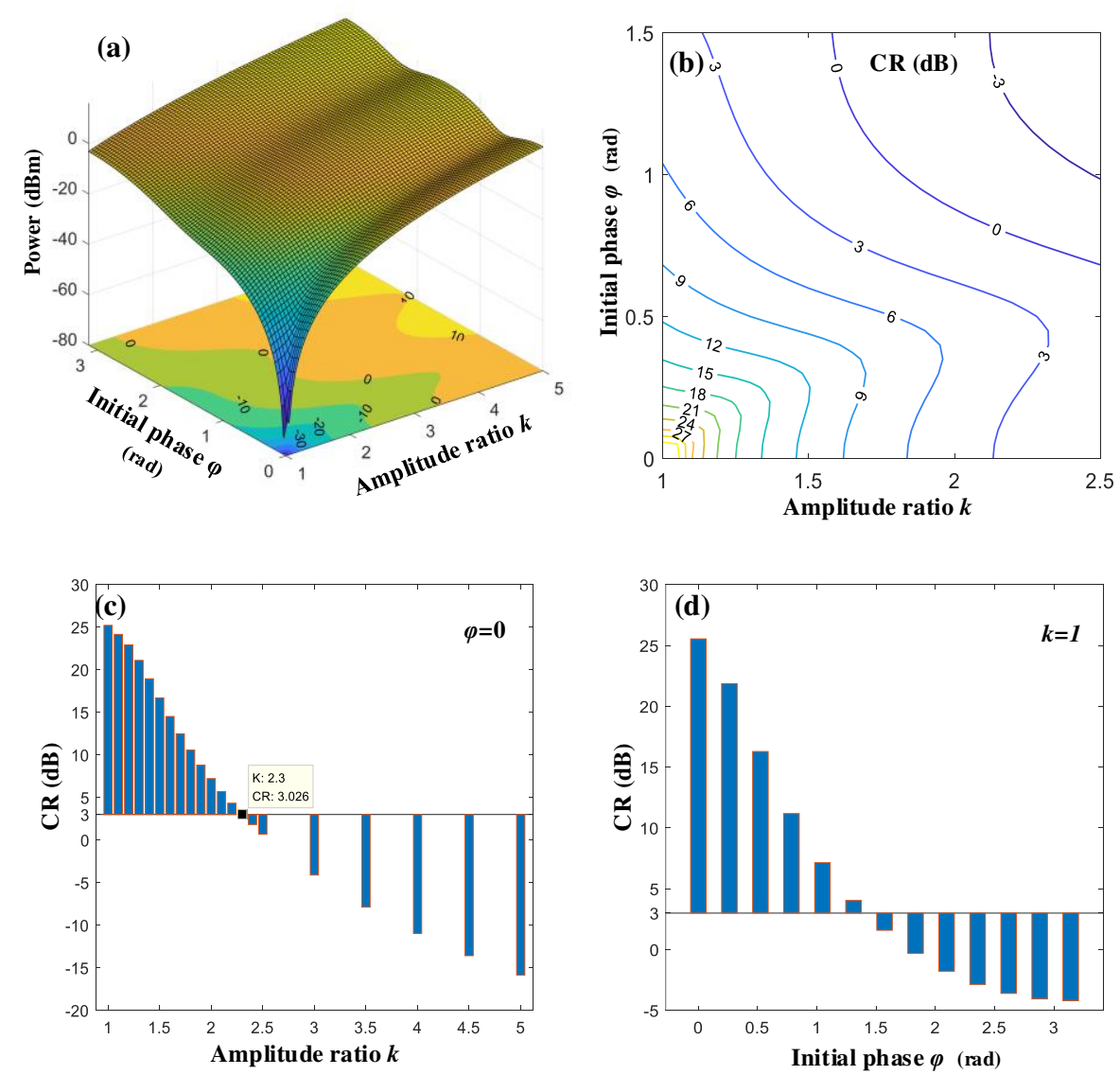

Fig. 7. (a) The theoretical relationship and contour among the power, amplitude ratio and initial phase. (b) The theoretical contour of CR. (c) Simulation results of the amplitude ratio and CR.(d) Simulation results of the initial phase and CR.

\subsection{Sensitivity and multi-frequency measurement of power cancellation}

In this work, the RFM is performed based on the interference of MZI, which is sensitive to the RF power and noise disturbance. In order to achieve a well-performed measurement, the RF power should be higher than $7 \mathrm{dBm}$. Nevertheless, in a real electronic warfare, the power of the intercepted RF signal is usually very low. In the scenario that requires the sensitivity, the method of power cancellation can be implemented to measure frequency. Fortunately, the frequency measurement based on power cancellation is determined by the notch position, it is not sensitive to power (optical power and microwave power) disturbance. Frequency identification can be achieved when the notch depth is greater than LDB. The measurement results of power cancellation at different RF powers are shown in Fig. 8. It can be figured that CR decreases with the reduced RF power. When the RF power decreases to $-13 \mathrm{dBm}$, the $\mathrm{CR}$ deteriorates to near $\mathrm{LDB}$, i.e. the sensitivity of power cancellation is about $-13 \mathrm{dBm}$. Meanwhile, the RF power has little influence on the measurement error, and the notch width is maintained at $4 \mathrm{MHz}$. 


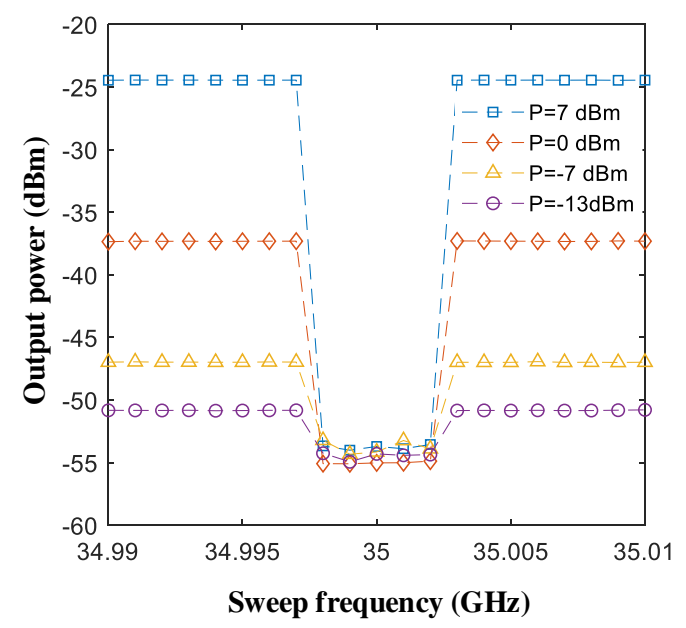

Fig. 8. Sensitivity measurement results of power cancellation.

The ACF established by the RFM is a frequency-to-power mapping frequency measurement method. Although it can realize instantaneous frequency measurement over a very wide range, only single-tone signal frequency can be measured. When the intercepted RF signal contains multiple frequencies, the scanning measurement based on power cancellation is required. Meanwhile, the minimum resolution of multi-frequency measurement is also investigated. The parameters setting of multi-frequency and resolution measurement are shown in Table 2.

Table 2. The parameters setting of multi-frequency and resolution measurement.

\begin{tabular}{cccc}
\hline & Frequency & Power & Initial phase (degree) \\
\hline RF1 & $2 \mathrm{GHz}$ & $0 \mathrm{dBm}$ & 0 \\
RF2 & $9 \mathrm{GHz}$ & $0 \mathrm{dBm}$ & 45 \\
RF3 & $12 \mathrm{GHz}$ & $-5 \mathrm{dBm}$ & 45 \\
RF4 & $18 \mathrm{GHz}$ & $-7 \mathrm{dBm}$ & 0 \\
RF5 \& RF6 & $5 \mathrm{GHz} \& 5.01 \mathrm{GHz}$ & $7 \mathrm{dBm}$ & 0 \\
Sweep signal & $0.5 \mathrm{GHz} 20 \mathrm{GHz}$ & $0 \mathrm{dBm}$ & 0 \\
\hline
\end{tabular}

The measured results of multi-frequency are shown in Fig .9(a). It can be figured that the power notches are be observed at $2 \mathrm{GHz}, 9 \mathrm{GHz}, 12 \mathrm{GHz}$ and $18 \mathrm{GHz}$, which means that the frequency measurement is well performed. Moreover, due to the various power and random phase of the RF signals, the depths of the power notches (CR) are also different. In addition, to investigate the resolution, the DPMZM2 is driven by a two-tone signal of 5 and $5.01 \mathrm{GHz}$. And the measured results are shown in Fig .9(b). It can be seen that when the frequency interval of the two signals exceeds $10 \mathrm{MHz}$, them can be well distinguished. 

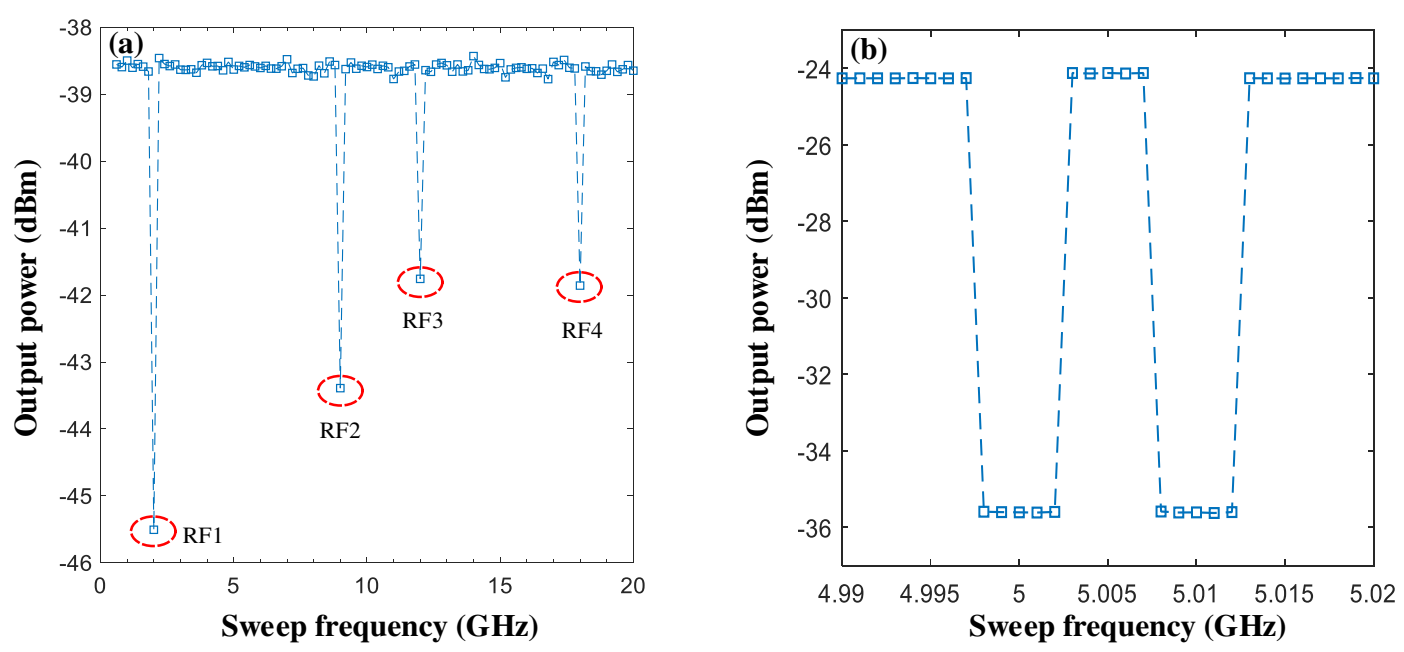

Fig. 9. (a) Simulation results of multi-frequency measurement. (b) The resolution results of the $5 \mathrm{GHz}$ and 5.01

GHz two-tone signal.

\section{Conclusion}

In conclusion, a high-accuracy photonics-assisted frequency measurement with rough-accurate compensation based on Mach-Zehnder interfering and power cancellation is proposed and demonstrated. The electro-optic modulation section is performed by an integrated PDM-DPMZM, which improves the stability and simplifies the system. The rough frequency measurement is firstly performed by the MZI-based fast frequency estimation. Thanks to the strict monotonous ACF, the measurement error of less than $0.3 \mathrm{GHz}$ is achieved over the range of $0.5 \mathrm{GHz} \sim 39 \mathrm{GHz}$. According to the rough measurement results, the accurate frequency measurement is implemented in a much narrower sweeping range. The main principle of the AFM is to cancel the unknown RF signal by the sweep signal and thereby causes the power notch. The position of the notch reflects the frequency information of the RF signal. The simulation results reveal that the measurement accuracy can be improved to $4 \mathrm{MHz}$, and the resolution of $10 \mathrm{MHz}$ is achieved. Moreover, this technique shows the broad range, well-performed accuracy and high resolution to implement multi-frequency identification. It has potential to be integrated into the future reconnaissance and detection receiver.

\section{Acknowledgements}

This research was supported in part by the National Natural Science Foundation of China under Grant 61901507, Grant 62001505, in part by the Natural Science Foundation of Shaanxi Province under Grant 2019JQ-707, and Grant 2020JQ-469.

\section{References}

[1] Tao Lin, Canwen Zou, Zhike Zhang, et al., Differentiator-Based Photonic Instantaneous Frequency Measurement for Radar Warning Receiver, Journal of Lightwave Technology, vol. 38, no. 15, pp. 3942-3949, Aug, 2020.

[2] Hossein Emami, Mohammadreza Hajihashemi, Sayed Ehsan Alavi, et al., Microwave photonics instantaneous frequency measurement receiver based on a Sagnac loop, Opt. Lett, vol. 43, no. 10, pp. 2233-2236, May, 2018. 
[3] C. Zhang, J. Zhang, and Z. Zhao, Microwave Instantaneous Frequency Measurement (IFM) Approach Based on an Integrated Photonic Ti:LiNbO 3 Y Branch, Current Optics and Photonics, vol. 4, no. 4, pp. 361-367, Aug. 2020.

[4] Danqi Feng, Heng Xie, Lifen Qian, Qinhong Bai, and Junqiang Sun, Photonic approach for microwave frequency measurement with adjustable measurement range and resolution using birefringence effect in highly non-linear fiber, Opt. Express vol. 23, no. 13, pp. 17613-17621, Jun. 2015.

[5] Jian D, Xu K. , Sun X. , et al. A Simple Photonic-Assisted Microwave Frequency Measurement System Based on MZI With Tunable Measurement Range and High Resolution. IEEE Photonics Technology Letters, vol. 22, no. 15, pp. 1162-1164, Aug. 2010.

[6] Yueqin Li, Li Pei, Jing Li, et al., Theory study on a range-extended and resolution-improved microwave frequency measurement, Journal of Modern Optics, vol. 63, no. 7, pp. 613-620, Sep. 2015.

[7] Guo Y, Ma J., Photonic instantaneous microwave frequency measurement with adjustable measurement range based on an electro-optical polarization modulator, Appl Opt, vol. 59, no. 7, pp. 1808-1816, Mar. 2020.

[8] Li X, Wen A, Ma X, Chen W, Gao Y, Zhang W, Tu Z, Xiang S., Photonic microwave frequency measurement with a tunable range based on a dual-polarization modulator, Appl Opt, vol. 55, no. 31, pp. 8727-8731, Nov. 2016.

[9] Yang Chen, Weifeng Zhang, Jingxuan Liu, and Jianping Yao, On-chip two-step microwave frequency measurement with high accuracy and ultra-wide bandwidth using add-drop micro-disk resonators, Opt. Lett, vol. 44, no. 10, pp. 2402-2405, May. 2019.

[10] A. O. J. Wiberg, et al., Coherent Filterless Wideband Microwave/Millimeter-Wave Channelizer Based on Broadband Parametric Mixers, Journal of Lightwave Technology, vol. 32, no. 20, pp. 3609-3617, Oct. 2014.

[11] X. Zou, W. Li, W. Pan, L. Yan and J. Yao, Photonic-Assisted Microwave Channelizer With Improved Channel Characteristics Based on Spectrum-Controlled Stimulated Brillouin Scattering, IEEE Transactions on Microwave Theory and Techniques, vol. 61, no. 9, pp. 3470-3478, Sep. 2013.

[12] Duan, Y., Liao, C., Zhou, H. et al. Ultrafast electrical spectrum analyzer based on all-optical Fourier transform and temporal magnification. Optics Express, vol. 25, no.7, pp.7520-7529, Apr. 2017.

[13] Tuan A. Nguyen, Erwin H. W. Chan, and Robert A. Minasian, Instantaneous high-resolution multiple-frequency measurement system based on frequency-to-time mapping technique, Opt. Lett, vol. 39, no. 8, pp. 2419-2422, 2014.

[14] Hao T, Tang J, Li W, Zhu N, Li M., Microwave photonics frequency-to-time mapping based on a Fourier domain mode locked optoelectronic oscillator, Optics Express, vol. 26, no. 26, pp. 3358233591, Dec. 2018.

[15] Tao Lin, Zhike Zhang, Canwen Zou, et al., Photonic multiple frequency measurement with phase identification based on frequency conversion, Optics Communications, vol. 474, 126060, May. 2020.

[16] J. Liu, T. Shi and Y. Chen, High-Accuracy Multiple Microwave Frequency Measurement With TwoStep Accuracy Improvement Based on Stimulated Brillouin Scattering and Frequency-to-Time Mapping, Journal of Lightwave Technology, vol. 39, no. 7, pp. 2023-2032, Apr. 2021.

[17] Hengyun Jiang, David Marpaung, Mattia Pagani, et al., Wide-range, high-precision multiple microwave frequency measurement using a chip-based photonic Brillouin filter, Optica, vol. 3, no. 1, pp. 30-34, Jan. 2016. 
[18] W. Jiao, K. You and J. Sun, Multiple Microwave Frequency Measurement With Improved Resolution Based on Stimulated Brillouin Scattering and Nonlinear Fitting, IEEE Photonics Journal, vol. 11, no. 1, pp. 1-12, Feb. 2019.

[19] Peng Li, Lianshan Yan, Jia Ye, et al., Photonic generation of binary and quaternary phase-coded microwave signals by utilizing a dual-polarization dual-parallel Mach-Zehnder modulator, Optics express, vol. 26, no. 21, pp. 28013-28021, 2018. 